\title{
Geospatial analysis, web-based mapping and determinants of prostate cancer incidence in Georgia counties: evidence from the 2012-2016 SEER data
}

Justice Moses Kwaku Aheto ( $\nabla$ justiceaheto@yahoo.com )

University of Ghana https://orcid.org/0000-0003-1384-2461

Ovie A. Utuama

University of South Florida

Getachew A. Dagne

University of South Florida

\section{Research}

Keywords: Prostate cancer, Geospatial modelling, Mapping prostate cancer, Disease mapping, R-INLA, SEER Program, Georgia, USA

Posted Date: June 17th, 2020

DOI: https://doi.org/10.21203/rs.3.rs-35902/v1

License: (c) (1) This work is licensed under a Creative Commons Attribution 4.0 International License. Read Full License

Version of Record: A version of this preprint was published at BMC Cancer on May 6th, 2021. See the published version at https://doi.org/10.1186/s12885-021-08254-0. 
Geospatial analysis, web-based mapping and determinants of prostate cancer incidence in Georgia counties: evidence from the 2012-2016 SEER data.

Authors:

Justice Moses K. Aheto ${ }^{1,2 *}$, Ovie A. Utuama ${ }^{2}$ and Getachew A. Dagne ${ }^{2}$

\section{Authors' Affiliations:}

${ }^{1}$ Department of Biostatistics, School of Public Health, College of Health Sciences, University of Ghana, Ghana.

${ }^{2}$ College of Public Health, University of South Florida, USA.

\section{*Corresponding author:}

Justice Moses K. Aheto

Department of Biostatistics

School of Public Health

College of Health Sciences

University of Ghana

P. O. Box LG13, Legon-Accra.

E-mail: justiceaheto@yahoo.com; jmkaheto@ug.edu.gh 


\begin{abstract}
Background: Prostate cancer ( $\mathrm{CaP}$ ) cases are high in the United States. According to the American Cancer Society, there are an estimated number of $174,650 \mathrm{CaP}$ new cases in 2019. The estimated number of deaths from $\mathrm{CaP}$ in 2019 is 31,620, making CaP the second leading cause of cancer deaths among American men with lung cancer been the first. Our goal is to estimate and map prostate cancer relative risk, with the ultimate goal of identifying counties at higher risk where interventions and further research can be targeted.
\end{abstract}

Method: The 2012-2016 Surveillance, Epidemiology, and End Results (SEER) Program data was used in this study. Analyses were conducted on 159 Georgia counties. The outcome variable is incident prostate cancer. We employed a Bayesian geospatial model to investigate both measured and unmeasured spatial risk factors for prostate cancer. We visualised the risk of prostate cancer by mapping the predicted relative risk and exceedance probabilities. We finally developed interactive web-based maps to guide optimal policy formulation and intervention strategies.

Results: Number of persons above age 65 years and below poverty, higher median family income, number of foreign born and unemployed were risk factors independently associated with prostate cancer risk in the non-spatial model. Except number of foreign born, all these risk factors were also significant in the spatial model with the same direction of effects. Substantial geographical variations in prostate cancer incidence were found in the study. The predicted mean relative risk was 1.20 with a range of 0.53 to 2.92. Individuals residing in Towns, Clay, Union, Putnam, Quitman, and Greene counties were at increased risk of prostate cancer incidence while those residing in Chattahoochee were at the lowest risk of prostate cancer incidence.

Conclusion: Our results can be used as an effective tool in the identification of counties that require targeted interventions and further research by program managers and policy makers as part of an overall strategy in reducing the prostate cancer burden in Georgia State and the United States as a whole.

\title{
Keywords
}

Prostate cancer, Geospatial modelling, Mapping prostate cancer, Disease mapping, R-INLA, SEER Program, Georgia, USA. 


\section{Background}

Prostate cancer is the leading diagnosis of malignancy and the second cause of mortality among American men, with an estimated national annual health care cost of $\$ 9.8$ billion $[1,2]$. The United States Cancer Statistics reported 192,443 new cases of prostate cancer in 2016, with an incidence rate of 101 per 100,000 men, and 30,370 prostate cancer deaths or 19 deaths per 100,000 during the same year [3]. Despite an overall decline in incidence across the United States since the early 1990s [4], there remain pockets of high prostate cancer burden.

In the United States, the state of Georgia has the second largest annual incidence rate of prostate cancer [3]. In 2016, there were 7,160 reported new cases and 889 deaths in the state, with associated incidence and mortality rates of 133 and 23 per 100,000 men, respectively [3]. African American (AA) men not only have higher incidence of prostate cancer but also demonstrate $60 \%$ more mortality than white men, after controlling for incidence [5]. As $32 \%$ of Georgia consists of AA [6], it represents an unusual opportunity to investigate community factors associated with a highrisk population. Although a few studies have identified high prostate cancer incidence in the southwest of the state $[7,8]$, the sociodemographic characteristics of these regions are not well described.

For the purpose of planning for prostate cancer interventions with limited health resources, it is important to characterize and identify predictors of high prostate cancer burden at the community level. The present study, therefore, aims to 1) model and map Georgia county incidence of prostate cancer, 2) evaluate county sociodemographic factors associated with high incidence of prostate cancer.

\section{Materials and Methods}

\section{Data source and study population}

We used the Surveillance, Epidemiology, End Results (SEER) population-based cancer registry, which is publicly available data to investigate county-level distribution of prostate cancer cases in the state of Georgia. Only newly diagnosed cases 40 years and older from January 1, 2012 through December 31, 2016 were used for this study, because case reporting to SEER from the greater Georgia area started in 2010 and at the time of analysis SEER's most current county attributes data spanned the 2012 to 2016 period. The greater Georgia area includes all counties in the state, except the 15 represented by the older Atlanta and Rural Georgia areas previously reported to SEER [9]. Therefore, since 2010 SEER captures cancer data from all 159 counties in Georgia. The SEER Georgia registry reports clinical, or preferentially pathologic diagnosis of cancer from eligible patient records in 
hospitals, laboratories and physician offices $[10,11]$. Patients must be Georgia residents at the time of diagnosis, even though the address of residence is not reported in the registry. Only patients with an International Classification of Diseases for Oncology, third edition, (ICD-O-3) with topography code C61 and behaviour code 3 were included for analysis. SEER, being one of the oldest registries in the country, represents the gold standard in reporting standards and data quality, with completeness rates of more than $97 \%$ [12-14].

SEER data are publicly available deidentified records of cancer cases. Permission was sort from the SEER Program to access and use the data for this study and was approved. We did not attempt to identify, contact patients or link records to identifiable health information.

\section{Outcome variable}

The outcome variable is the number of incident prostate cancer cases per county. Detailed information is provided under the statistical analysis section.

\section{Covariates}

The covariates used in this study are those that were identified in the literature to be associated with the prostate cancer incidence $[2,15-17]$. These include proportion of blacks, number of persons above age 65 years, having at least a bachelor's degree, age at diagnosis, number below poverty, number of foreign born, percentage rural population, median family income, and number of unemployed.

\section{Statistical analysis}

We employed a Bayesian geospatial model to investigate both measured and unmeasured spatial risk factors for prostate cancer among men residing in 159 counties in Georgia State.

\section{Model Formulation}

Let $Y_{i}$ be the observed counts of prostate cancer cases in county $i$ and $E_{i}$ be the expected number of prostate number cases in county $i$. We implement Besag-York-Mollié (BYM) model [18], a wellknown spatial model in disease mapping applications.

We assumed that $Y_{i}$ are conditionally independently Poisson distributed and modelled the $Y_{i}$ as:

$$
Y_{i} \sim \operatorname{Poisson}\left(E_{i} \theta_{i}\right), i=1,2, \ldots, n
$$

where $n$ is the number of counties (i.e $\mathrm{n}=159$ ) and $\theta_{i}$ is the relative risk in county $i$. We expressed the logarithm of $\theta_{i}$ as:

$$
\log \left(\theta_{i}\right)=\beta_{0}+\boldsymbol{d}\left(\boldsymbol{x}_{\boldsymbol{i}}\right)^{\prime} \beta+u_{i}+v_{i}
$$


where $\beta_{0}$ is the intercept parameter that represents the overall risk, $d($.$) is a vector of observed$ covariates, $\beta$ is a vector of regression coefficients for the covariates, $u_{i}$ is a spatial structured effect component modelled with a conditional autoregressive (CAR) distribution given as

$u_{i} \mid \boldsymbol{u}_{-\boldsymbol{i}} \sim N\left(\bar{u}_{\delta_{i}}, \frac{\sigma_{u}^{2}}{n \delta_{i}}\right)$, and $v_{i}$ is an unstructured spatial effect defined as $v_{i}=N\left(0, \sigma_{v}^{2}\right)$.

The relative risk $\theta_{i}$ quantifies whether county $i$ has higher $\left(\theta_{i}>1\right)$ or lower $\left(\theta_{i}<1\right)$ risk than the average risk in the reference population. We produced the probabilities of predicted relative risk being greater than a given threshold $c$ (exceedance probabilities, i.e. $P\left(\theta_{i}>c\right)$ ).

Finally, we visualised the risk of prostate cancer by mapping the predicted relative risk and exceedance probabilities. We developed interactive web-based maps to guide optimal policy formulation and intervention strategies targeted at improving the survival of prostate cancer patients and the overall health of men in Georgia.

To implement our model, the Integrated Nested Laplace Approximation (INLA) approach [19] with Stochastic Partial Differential Equation (SPDE) strategy was used under Bayesian framework [20]. All the analyses were implemented in R-INLA package $[21,22]$. To declare statistical significance, a pvalue $<0.05$ was considered.

\section{Results}

\section{Sample characteristics}

We present summary results of risk factors considered in the study and interpret the results based on the median and its associated interquartile range (IQR) due to non-normal distribution of most variables. On average, $33 \%(26)$ of the study participants were Blacks and the median number of persons having at least bachelor's degree was 1492(843). The median number of persons aged $\geq 65$ years was 1554(454) while the median age at diagnostic was $67(1.68)$. The median number of persons below poverty and foreign born were $2102(934)$ and 318(360) respectively, and with an average of $65 \%$ (39) rural population. On average, the median family income was $\$ 4,852(1118)$ and the median number of unemployed was 862(347) (Table 1). 
Table 1 Summary of risk factors considered in the study

\begin{tabular}{lcccc}
\hline \multicolumn{1}{c}{ Variables } & Median(IQR) & Mean(SD) & minimum & Maximum \\
\hline Proportion of blacks & $0.33(0.26)$ & $0.32(0.18)$ & 0 & 0.71 \\
Number of persons above age 65 years & $1554(454)$ & $1564(407)$ & 385 & 3312 \\
Number having at least a bachelor's degree & $1492(843)$ & $1757(870)$ & 494 & 4981 \\
Age at diagnosis (years) & $67(1.68)$ & $67(1.47)$ & 63 & 70 \\
Number below poverty & $2102(934)$ & $2174(685)$ & 642 & 4141 \\
Number of foreign born & $318(360)$ & $460(438)$ & 10 & 2913 \\
Percentage rural population & $65(39)$ & $60(29)$ & 0.25 & 100 \\
Median family income & $4852(1118)$ & $5097(1245)$ & 2250 & 10392 \\
Number of unemployed & $862(347)$ & $911(304)$ & 320 & 1894 \\
\hline
\end{tabular}

IQR: Interquartile Range; SD: Standard Deviation.

\section{Risk factors from non-spatial and spatial models}

Number of persons above age 65 years and below poverty, higher median family income, number of foreign born and unemployed were risk factors independently associated with prostate cancer risk in the non-spatial model (Figure 1).
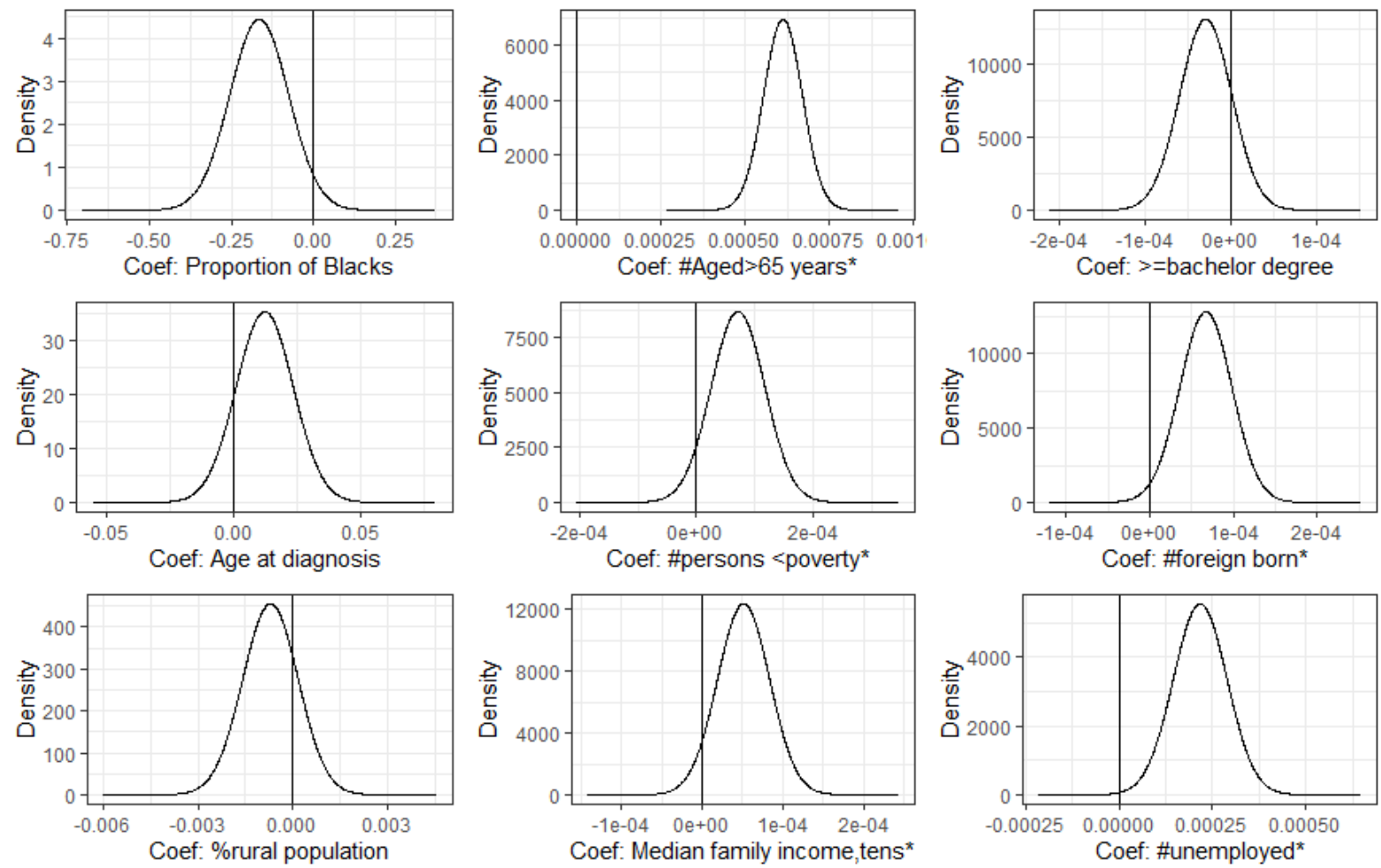
Figure 1 Risk factors associated with prostate cancer incidence in the non-spatial model

Except for number of foreign born, all these significant risk factors in the non-spatial model were also significant in the spatial model with the same direction of effects (Figure 2).
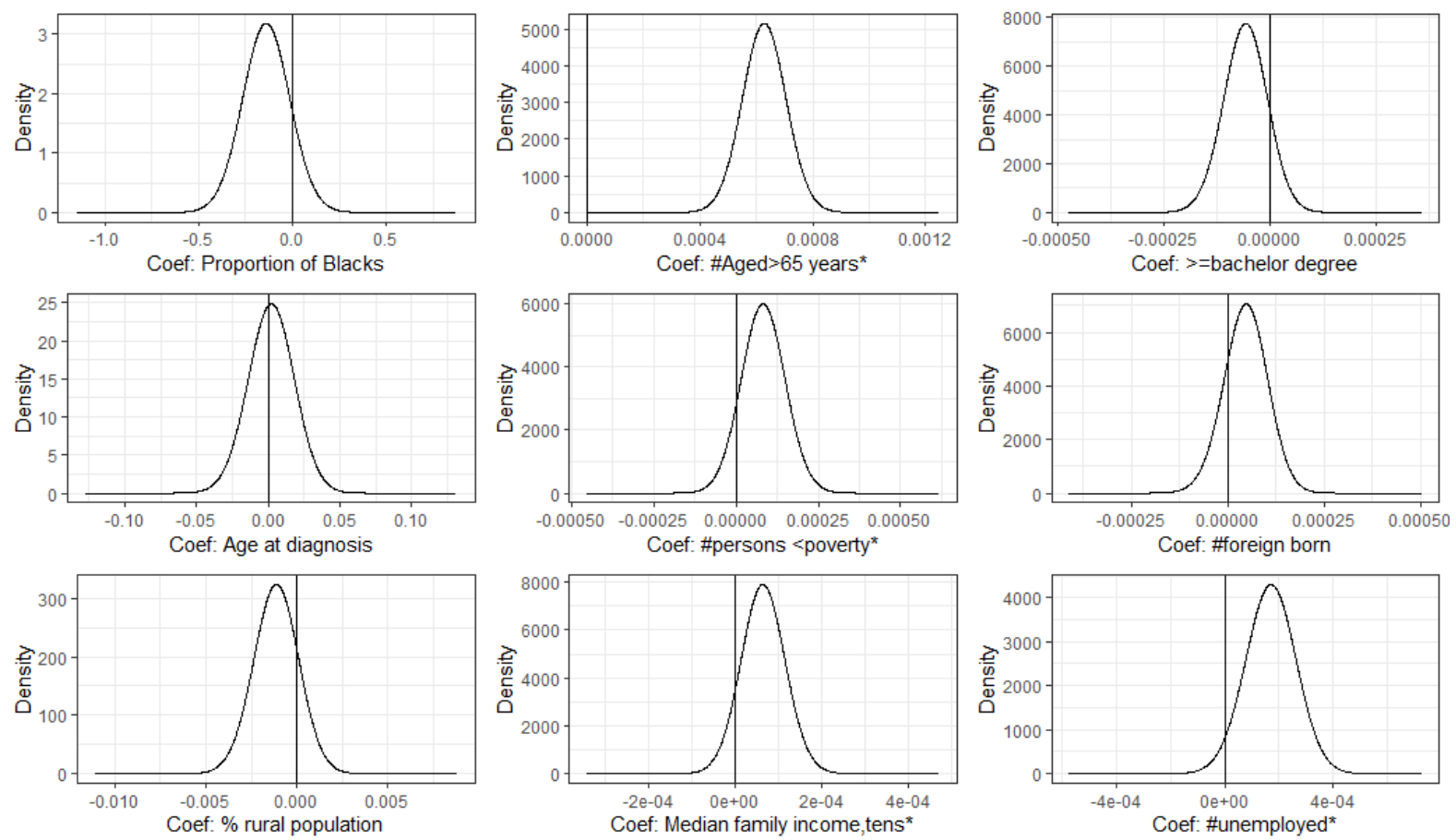

Figure 2 Risk factors associated with prostate cancer incidence in the spatial model

\section{Mapping predicted risk of prostate cancer incidence from the Bayesian spatial model}

Substantial geographical variations in prostate cancer incidence were found in the study (Figure 3). In addition, we presented the web-based interactive map of Figure 3 in the supplementary material online. The predicted mean relative risk (RR) was 1.20 with a range of $0.53(95 \% \mathrm{Cl}: 0.34,0.78)$ to 2.92 (95\% Cl: 2.13, 3.86). Individuals residing in Towns, Clay, Union, Putnam, Quitman, and Greene counties were at increased risk of prostate cancer incidence while those residing in Chattahoochee were at the lowest risk of prostate cancer incidence. 


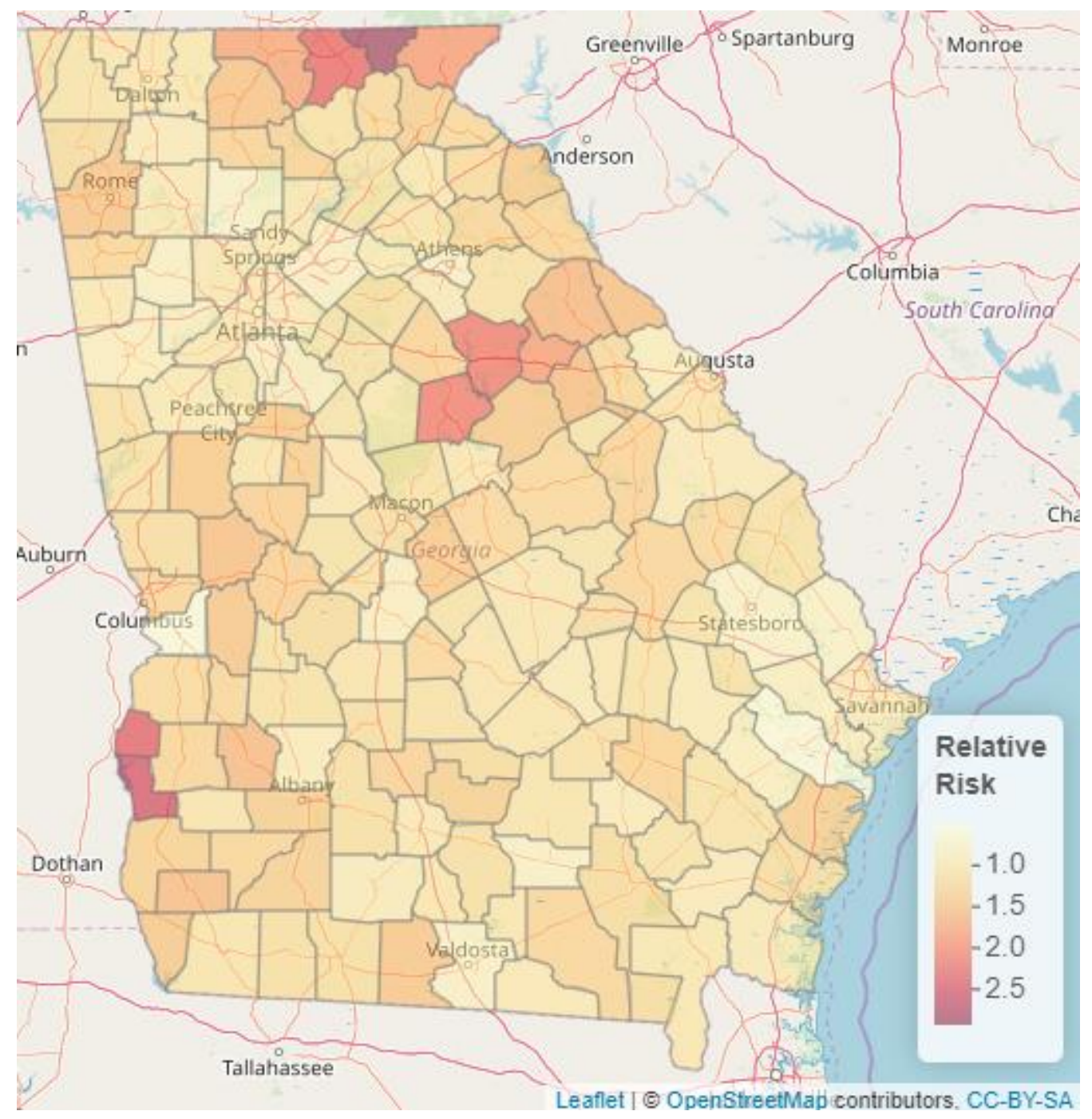

Figure 3 Spatial distribution of predicted prostate cancer relative risk in the Georgia State

Presented in Figures 4 and 5 are the predictive maps of the probability that the relative risk will exceed 1.5 and 2 respectively at a given county in the Georgia State. We also presented the webbased interactive map of Figures 4 and 5 in the supplementary material online. The deep red regions represent counties where the probability of the relative risk exceeding 1.5 (Figure 4) and 2 (Figure 5) are high. 


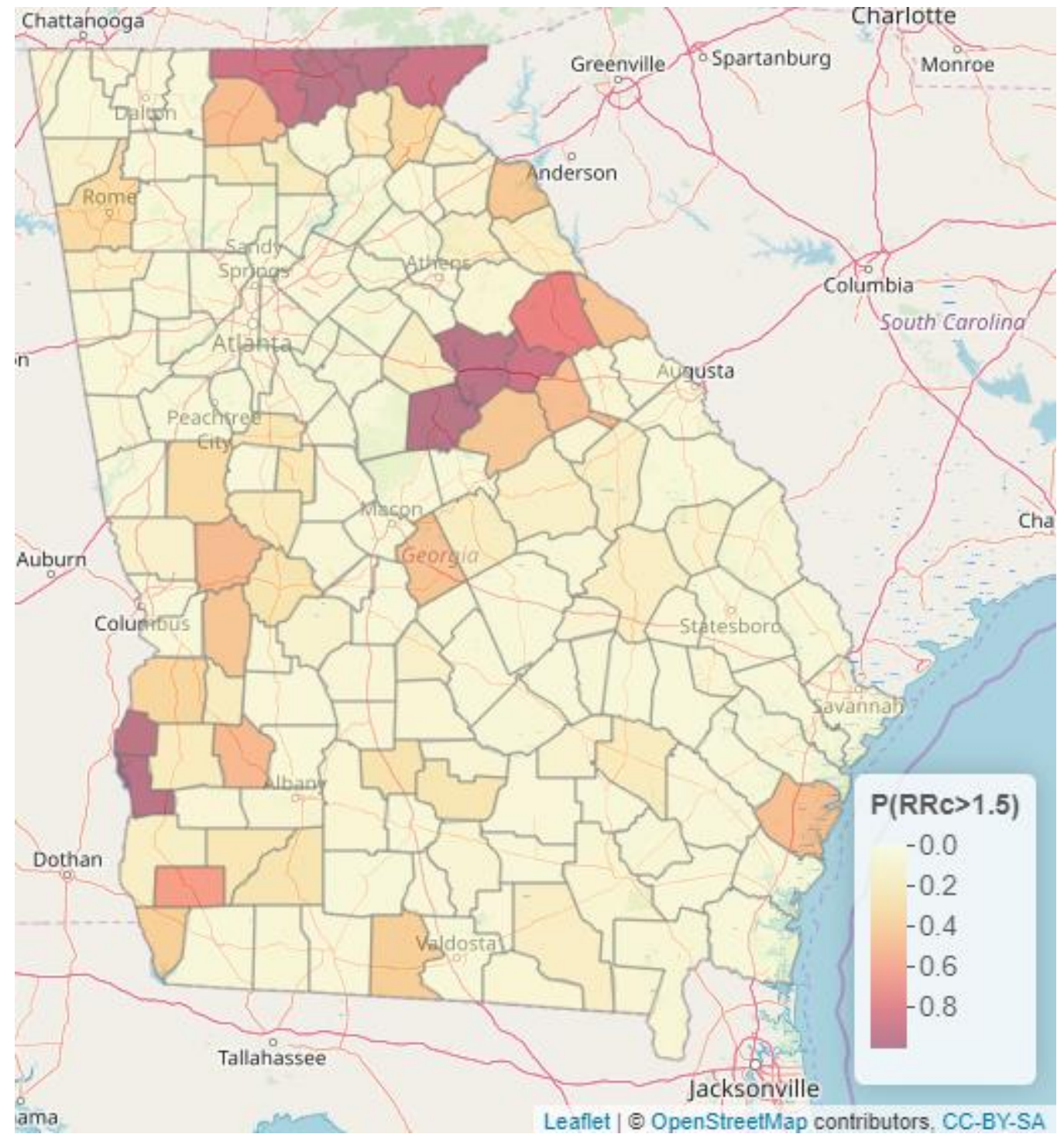

Figure 4 Predictive maps for exceedance probability of relative risk of 1.5 (i.e. $P(R R>1.5)$ )

The probability that the relative risk will exceed 1.5 is highest in Union, Towns, Putnam, Greene and Quitman counties (Figure 4). Also, the probability that the relative risk will exceed 2 is highest in Towns county with a probability of 0.99 (Figure 5). 


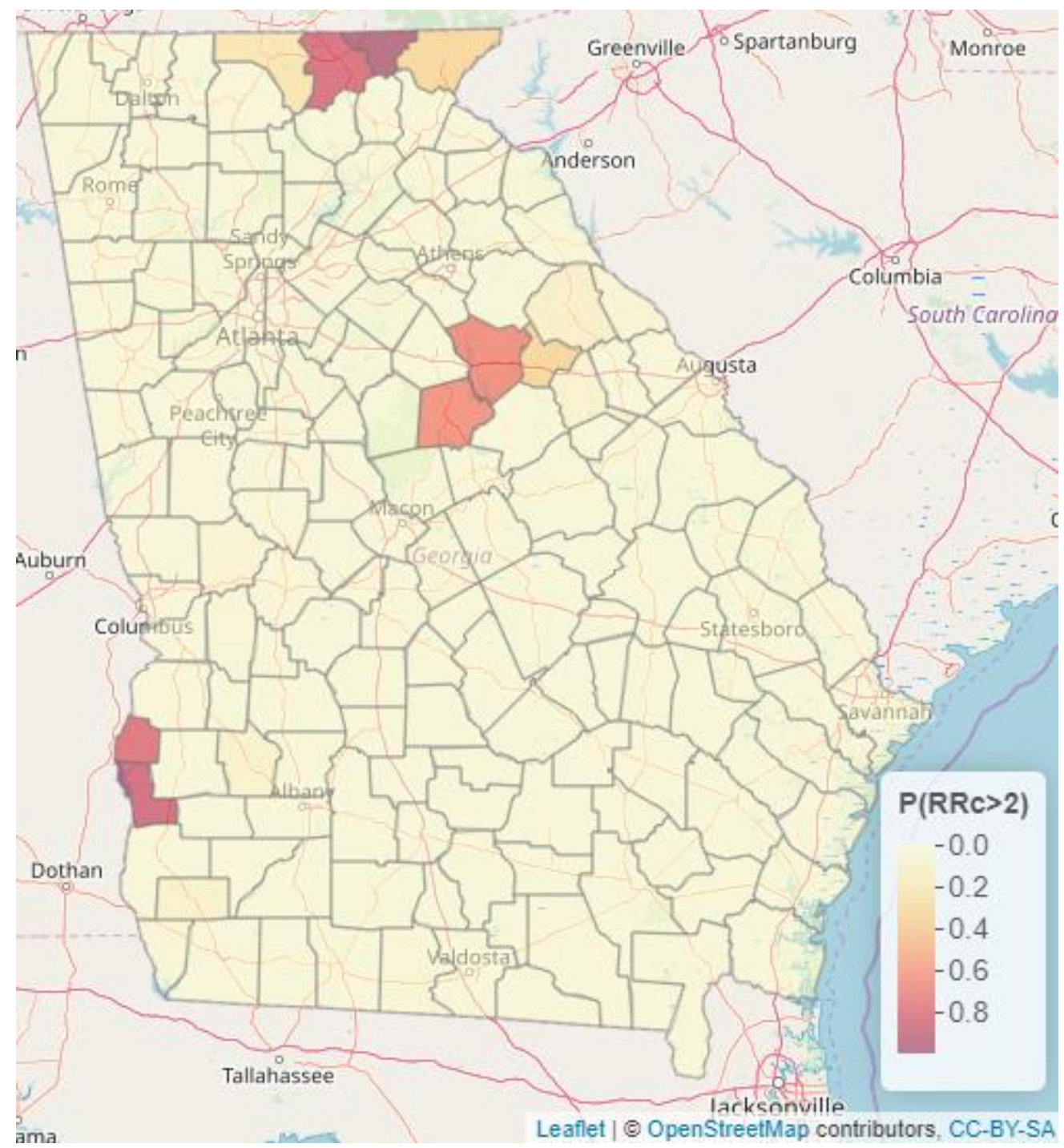

Figure 5 Predictive maps for exceedance probability of relative risk of 2 (i.e. $P(R R>2)$ )

\section{Discussion}

The study sets out to use Bayesian geospatial methods to model and map prostate cancer incidence in Georgia counties, and to evaluate county sociodemographic factors associated with high incidence of prostate cancer for the purpose of optimal planning for prostate cancer interventions amidst limited public health resources. Critical risk factors for prostate cancer identified in the present study include number of persons above age 65 years and below poverty, median family income, being a foreign born and unemployed. In contrast to previous studies [5,7], our study did not find an association between prostate cancer incidence and proportions of blacks and rural population. 
One of the important aims of this study is identification of high-risk counties for public health interventions amidst limited public health resources. This is critical because residential location of people could act as a marker for the socioeconomic, personal, and climatic/environmental factors that influence access to healthcare services and the general health of the people. Thus, spatial modelling and mapping provides the required tools to obtain an improved understanding of health outcomes of people by place for targeted public health interventions [7, 23-27]. The predicted relative risk ranges from $0.53(95 \% \mathrm{Cl}: 0.34,0.78)$ in Chattahoochee to $2.92(95 \% \mathrm{Cl}: 2.13,3.86)$ in Towns with a mean of 1.20. The study identified Towns (2.92) as the county with the highest prostate cancer incidence. Other counties with relatively high incidence include Clay $(R R=2.55)$, Quitman ( $R R=2.39)$, Union ( $R R=2.30)$, Greene $(R R=2.14)$ and Putnam $(R R=2.13)$ counties were at increased risk of prostate cancer incidence.

On closer examination of high risk prostate cancer counties, we observed that despite being predominantly white and better educated ( $25.1 \%$ with a Bachelor's degree) the main driver of risk in Towns County in the north of Georgia was its older population, reporting the largest proportion of persons at least 65 years of age (33.1\%). While advancing age is a well-known risk factor for prostate cancer, Clay and Quitman Counties in the southwest with large proportions of reported AfricanAmerican/black cases ( $52.2 \%$ and $46.5 \%$, respectively) also suggest that low educational attainment (7.4\% and $8.5 \%$ with a Bachelor's degree), high unemployment (18.9\% and $18.5 \%)$ and individual (39.8\% and $25.6 \%)$ and family poverty (28.7\% and $24.2 \%)$ may be additional risk factors in black communities. Exactly how these socioeconomic indices may impact prostate cancer risk within older black populations is not well known, but high cigarette use and alcohol consumption as well as poor diet have been hypothesized to mediate or moderate this risk [28]. Additional risk factors of exposures to water, air and soil pollution from agricultural farming of cash crops such as cotton, from the southwest through to central Georgia, may also be involved [29]. As neighbouring lower risk counties with large or predominantly black populations likely shared these environmental conditions with Clay and Quitman, our modelling suggests that prostate cancer risk in both communities is multifactorial, resulting from a possible confluence of negative lifestyle, economic and environmental factors experienced over long periods of time. Putnam and Greene Counties in central Georgia exemplified this aggregation of risk factors, demonstrating above average risk in many socioeconomic county characteristics. 
In comparing the high-risk counties with Chattahoochee and rural low-risk counties, we observed that population age was the single most obvious distinction. Low risk counties had a smaller proportion of elderly persons, irrespective of whether they were classified as rural. Chattahoochee had the youngest population (3.8\% 65 years and older), the highest educational attainment $(30 \%$ with a Bachelor's degree) and a large proportion of African-American prostate cancer cases (55.2\%), despite having a black population of $18.8 \%$ and 38 reported cases during the study period. Better education and lower poverty indices may have helped overcome typical health access barriers and explained, in part, the unusual proportion of incident black prostate cancer cases in this community. It is noteworthy that counties outside the Atlanta metro area with more than a $30 \%$ burden of black prostate cancer patients tended to be smaller in size than their predominantly white prostate cancer reporting counties, a finding that may explain the absence of an association between prostate cancer risk and proportion of blacks in counties.

Our study supports the findings of others that reported geographical differences in health outcomes such as prostate and lung cancers, malaria, malnutrition, mortality among others [5, 7, 23-25, 30]. Against the backdrop of a national reduction in incident prostate cancer, there remain pockets of high risk in the north, central and southwest areas of Georgia. The present study suggests that there may be racial differences in prostate cancer risk within counties. The aging population may be the main risk factor in overwhelmingly white counties while limited education and poverty may play a larger role in black counties. Importantly, this is an ecological study and the associations discussed herein should not be regarded as causal or necessarily significant at the level of individual prostate cancer patients.

\section{Strengths and limitation}

The use of Bayesian spatial analysis methods in this study provided an essential tool for the investigation of prostate cancer incidence in relation to risk factors to help in the better understanding of spatial distribution and potential etiologic mechanism of prostate cancer disease using an internationally recognised gold standard SEER data. Our modelling approach also allowed counties with small counts to borrow information from their neighbouring counties thereby reducing the risk of inflated relative risk due to small expected counts. Furthermore, unlike the frequentist spatial modelling approach, our Bayesian spatial modelling approach allowed graphical presentation of the posterior distribution of risk factor effects on the prostate cancer incidence as presented in Figures 1-2. The present study might have left out some potential risk factors that might explain 
some of the geographical differences in prostate cancer disease observed in the study so the findings should be interpreted with caution.

Our findings broadly support previous studies $[2,15-17,31]$ that report that older ages ( $\geq 65$ years), income (number below poverty and median family income), race (being a foreign born) and unemployed are critical risk factors for prostate cancer disease. For example, the finding that the number of persons aged 65 years or older increased the risk of the disease supports previous studies that reported that prostate cancer risk increases with age, and with incidence rate over 60\% [31-33]. The finding that increased number of foreign born increases the risk of prostate cancer disease supports previous studies that reported prostate cancer inequality by race [7].

\title{
Conclusion
}

Our modelling approach captured variation in prostate cancer risk over the whole of the Georgia State. The risk maps indicate substantial geographical variations in the risk of prostate cancer. This can be used as an effective tool in the identification of counties that require targeted interventions and further research by program managers and implementers as part of an overall strategy in reducing the prostate cancer burden in the Georgia State and the U.S. as a whole. For example, a further research could aim at identifying as yet unidentified risk factors that might have accounted for the geographical differences we observed in the prostate cancer disease among the counties in the Georgia State after we have accounted for the present risk factors in our model. Furthermore, we advocate for implementation of focused strategies to decrease prostate cancer incidence and to improve survival in the presence of the identified critical risk factors in this study.

\section{Abbreviations}

\author{
AA: African American \\ $\mathrm{Cl}$ : Credible Interval \\ ICD-O-3: International Classification of Diseases for Oncology, third edition \\ INLA: Integrated Nested Laplace Approximation \\ RR: Relative Risk \\ SEER: Surveillance, Epidemiology, and End Results \\ SPDE: Stochastic Partial Differential Equation \\ U.S.: United States of America
}




\section{Author contributions}

JMKA developed the concept. JMKA and OAU secured the data. JMKA analysed the data and wrote the first draft manuscript. JMKA, OAU and GAD contributed to the writing and reviewing of the various sections of the manuscript. All the authors reviewed the final version of the manuscript before submission. All authors read and approved the final manuscript.

\section{Declarations}

\section{Competing interest}

The authors declare that they have no competing interests.

\section{Acknowledgements}

This Fellowship was supported by the University of Ghana Building a New Generation of Academics in Africa (BANGA-Africa) Project with funding from the Carnegie Corporation of New York. The statements made and views are solely the responsibility of the authors. We are also grateful to the Surveillance, Epidemiology, and End Results (SEER) Program for making the data available for the study.

\section{Consent for publication}

Not applicable

\section{Funding}

Funding is not applicable to this paper. As a corresponding author, I have full access to all the data in the study and had final responsibility for the decision to submit for publication.

\section{Availability of data and material}

Data is freely available upon making official request to Surveillance, Epidemiology, and End Results (SEER) Program through the website at https://seer.cancer.gov/.

\section{Ethics approval and consent to participate}

Not applicable.

\section{List of Figures}

Figure 1 Risk factors associated with prostate cancer incidence in the non-spatial model

Figure 2 Risk factors associated with prostate cancer incidence in the spatial model

Figure 3 Spatial distribution of predicted prostate cancer relative risk in the Georgia State

Figure 4 Predictive maps for exceedance probability of relative risk of 1.5 (i.e. $P(R R>1.5)$ )

Figure 5 Predictive maps for exceedance probability of relative risk of 2 (i.e. $P(R R>2)$ ) 


\section{References}

1. Roehrborn CG, Black LK: The economic burden of prostate cancer. BJU International 2011, 108(6):806-813.

2. Siegel RL, Miller KD, Jemal A: Cancer statistics, 2019. CA: A Cancer Journal for Clinicians 2019, 69(1):7-34.

3. CDC:Centers for Disease Control and Prevention: United States Cancer Statistics (available online at https://gis.cdc.gov/Cancer/USCS/DataViz.html, accessed on 29th November 2019). In.: CDC; 2019.

4. Kelly SP, Rosenberg PS, Anderson WF, Andreotti G, Younes N, Cleary SD, Cook MB: Trends in the Incidence of Fatal Prostate Cancer in the United States by Race. Eur Urol 2017, 71(2):195-201.

5. Wagner SE, Hurley DM, Hébert JR, McNamara C, Bayakly AR, Vena JE: Cancer mortality-toincidence ratios in Georgia: describing racial cancer disparities and potential geographic determinants. Cancer 2012, 118(16):4032-4045.

6. USCB: United States Census Bureau: State and County Quick Facts: Georgia (available online at https://www.census.gov/quickfacts/GA, accessed on 4th March 2020. In.: USCB; 2020.

7. Wagner SE, Bauer SE, Bayakly AR, Vena JE: Prostate cancer incidence and tumor severity in Georgia: descriptive epidemiology, racial disparity, and geographic trends. Cancer Causes Control 2013, 24(1):153-166.

8. McNamara C, Davis V, Bayakly AR, Moon T: Prostate Cancer in Georgia, 2002-2006. In.: Georgia Department of Community Health, Division of Public Health, Chronic Disease, Healthy Behaviors, and Injury Epidemiology; 2020.

9. SEER: Surveillance Epidemiology and End Results Program: Georgia Center for Cancer Statistics (available online at https://seer.cancer.gov/registries/georgia.html, accessed on 3rd March 2020). In.: SEER; 2020.

10. Ruhl J, Adamo M, Dickie L: SEER Program Coding and Staging Manual 2016: Section V. In. Bethesda, MD 20850-9765: National Cancer Institute; 2016.

11. Scosyrev E, Messing J, Noyes K, Veazie P, Messing E: Surveillance Epidemiology and End Results (SEER) program and population-based research in urologic oncology: an overview. Urol Oncol 2012, 30(2):126-132.

12. Duggan MA, Anderson WF, Altekruse S, Penberthy L, Sherman ME: The Surveillance, Epidemiology, and End Results (SEER) Program and Pathology: Toward Strengthening the Critical Relationship. The American journal of surgical pathology 2016, 40(12):e94-e102.

13. Park HS, Lloyd S, Decker RH, Wilson LD, Yu JB: Overview of the Surveillance, Epidemiology, and End Results database: evolution, data variables, and quality assurance. Curr Probl Cancer 2012, 36(4):183-190.

14. Zippin C, Lum D, Hankey BF: Completeness of hospital cancer case reporting from the SEER Program of the National Cancer Institute. Cancer 1995, 76(11):2343-2350.

15. ACS: American Cancer Society: Cancer Statistics Center: Georgia [Date accessed: 23/08/2019, available at https://cancerstatisticscenter.cancer.org/\#!/state/Georgia]. In.: ACS; 2019.

16. Lund Nilsen $\mathrm{TI}$, Johnsen $\mathrm{R}$, Vatten $\mathrm{L}$ : Socio-economic and lifestyle factors associated with the risk of prostate cancer. Br J Cancer 2000, 82(7):1358-1363.

17. Hastert TA, Beresford SA, Sheppard L, White E: Disparities in cancer incidence and mortality by area-level socioeconomic status: a multilevel analysis. J Epidemiol Community Health 2015, 69(2):168-176. 
18. Besag J, York J, Mollié A: Bayesian Image Restoration with Applications in Spatial Statistics (with Discussion) Annals of the Institute of Statistical Mathematics 1991, 43:1-59.

19. Rue H, Martino S, Chopin N: Approximate Bayesian inference for latent Gaussian models by using integrated nested Laplace approximations. J Roy Stat Soc B Stat Meth 2009, 71.

20. Lindgren F, Rue H, Lindström J: An explicit link between Gaussian fields and Gaussian Markov random fields: the stochastic partial differential equation approach. Journal of the Royal Statistical Society: Series B (Statistical Methodology) 2011, 73(4):423-498.

21. Lindgren F, Rue H: Bayesian Spatial Modelling with R-INLA. Journal of Statistical Software; Vol 1, Issue 19 (2015) 2015.

22. Rue H, Martino S, Lindgren F, Simpson D, Riebler A, Krainski E: INLA: Functions Which Allow to Perform a Full Bayesian Analysis of Structured Additive Models Using Integrated Nested Laplace Approximaxion. R package version 00-1404466487, URL http://wwwR-INLAorg 2014.

23. Aheto JMK, Taylor BM, Keegan TJ, Diggle PJ: Modelling and forecasting spatio-temporal variation in the risk of chronic malnutrition among under-five children in Ghana. Spat Spatiotemporal Epidemiol 2017, 21:37-46.

24. Aheto JMK: Predictive model and determinants of under-five child mortality: evidence from the 2014 Ghana demographic and health survey. BMC Public Health 2019, 19(1):64.

25. Diggle P, Moyeed R, Rowlingson B, Thomson M: Childhood malaria in the Gambia: A casestudy in model-based geostatistics. In: Journal of the Royal Statistical Society: Series $C$ (Applied Statistics). vol. 51. Series C (Applied Statistics); 2002: 493-506.

26. Kandala NB, Madungu TP, Emina JB, Nzita KP, Cappuccio FP: Malnutrition among children under the age of five in the Democratic Republic of Congo (DRC): does geographic location matter? BMC Public Health 2011, 11:261.

27. Nykiforuk Cl, Flaman LM: Geographic information systems (GIS) for Health Promotion and Public Health: a review. Health Promot Pract 2011, 12(1):63-73.

28. Brotherton L, Welton M, Robb SW: Racial disparities of pancreatic cancer in Georgia: a county-wide comparison of incidence and mortality across the state, 2000-2011. Cancer Med 2016, 5(1):100-110.

29. Blomme C, Roubal A, Givens M, Johnson S, Brown L: Georgia County Health Rankings State Report 2020. [Available at: https://www.countyhealthrankings.org/reports/statereports/2020-georgia-report, accessed 10th May 2020]. In.: University of Wisconsin Population Health Institute; 2020.

30. Mokdad AH, Dwyer-Lindgren L, Fitzmaurice C, Stubbs RW, Bertozzi-Villa A, Morozoff C, Charara R, Allen C, Naghavi M, Murray CJL: Trends and Patterns of Disparities in Cancer Mortality Among US Counties, 1980-2014. JAMA 2017, 317(4):388-406.

31. Gann PH: Risk factors for prostate cancer. Reviews in urology 2002, 4 Suppl 5(Suppl 5):S3S10.

32. Rawla P: Epidemiology of Prostate Cancer. World journal of oncology 2019, 10(2):63-89.

33. Merriel SWD, Funston G, Hamilton W: Prostate Cancer in Primary Care. Advances in Therapy 2018, 35(9):1285-1294. 
Figures
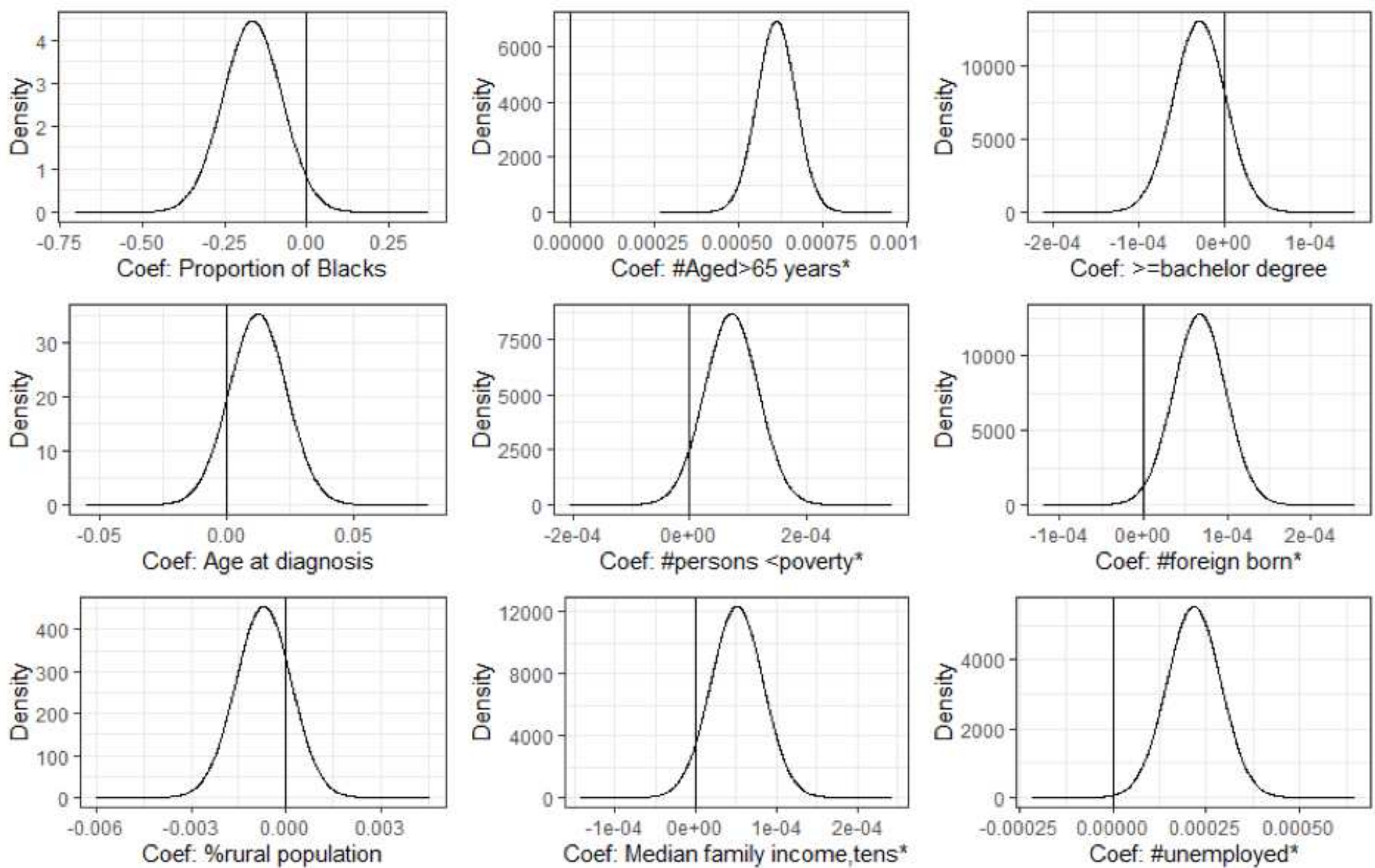

Figure 1

Risk factors associated with prostate cancer incidence in the non-spatial model 

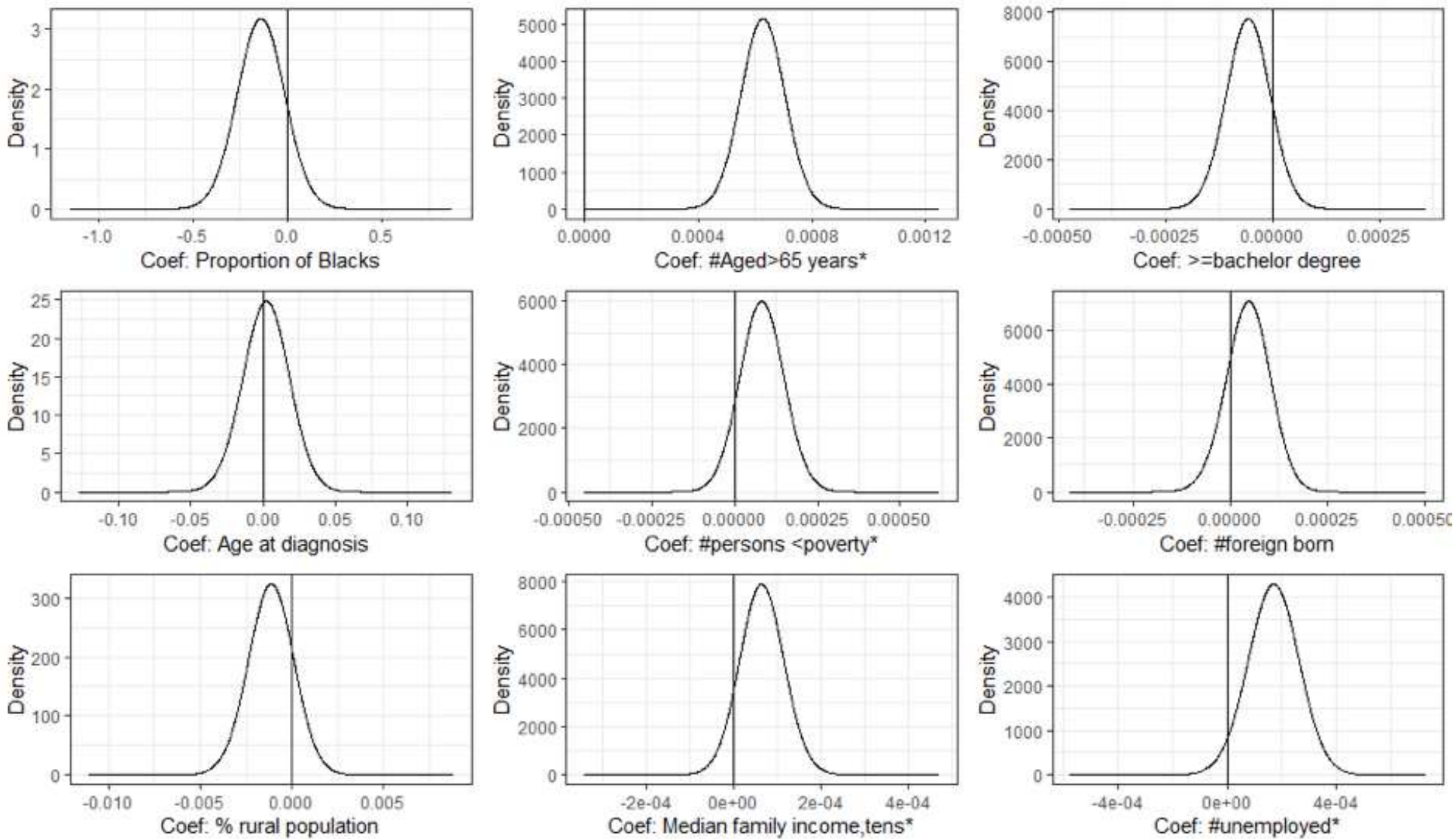

Figure 2

Risk factors associated with prostate cancer incidence in the spatial model 


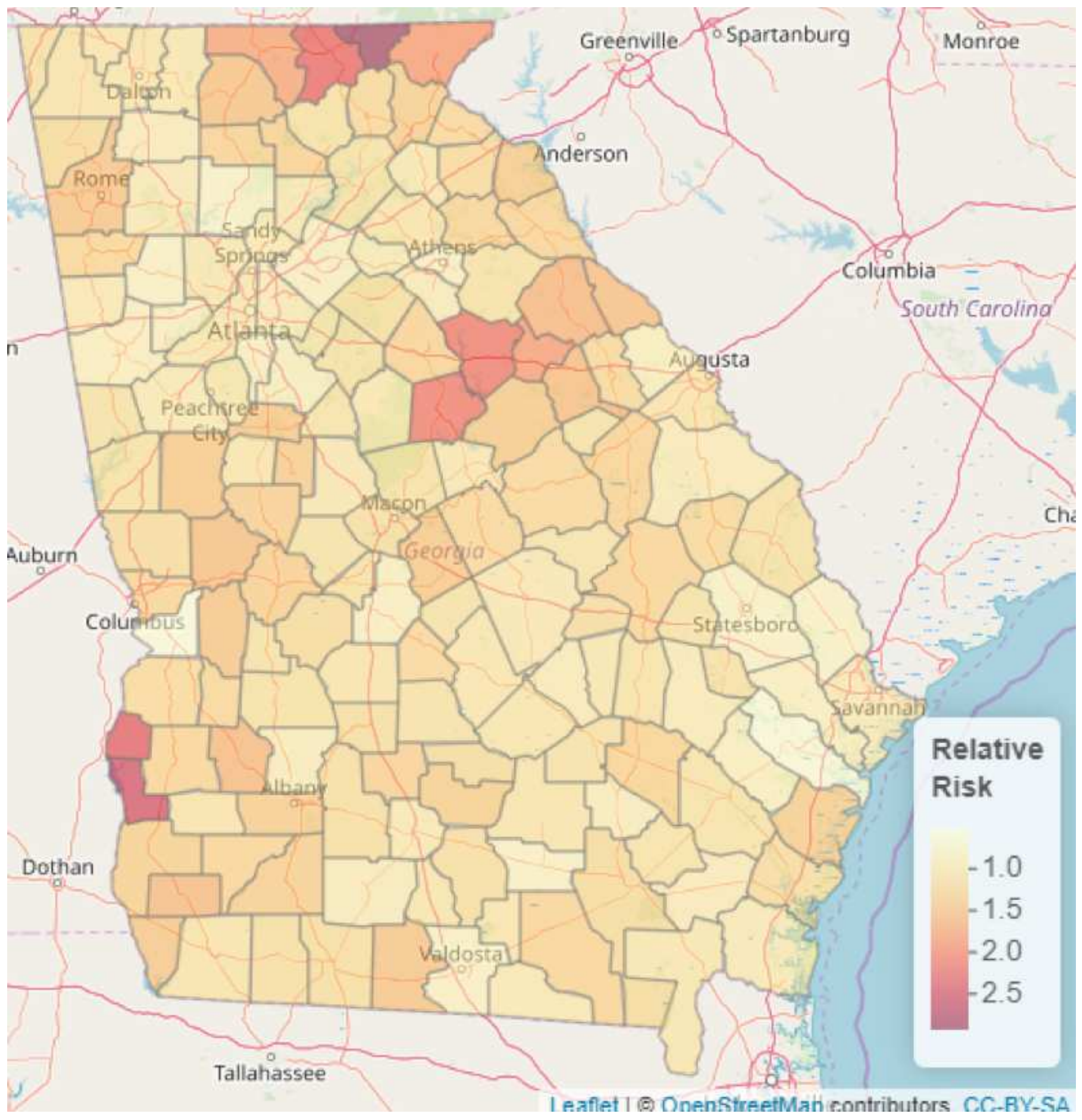

Figure 3

Spatial distribution of predicted prostate cancer relative risk in the Georgia State 


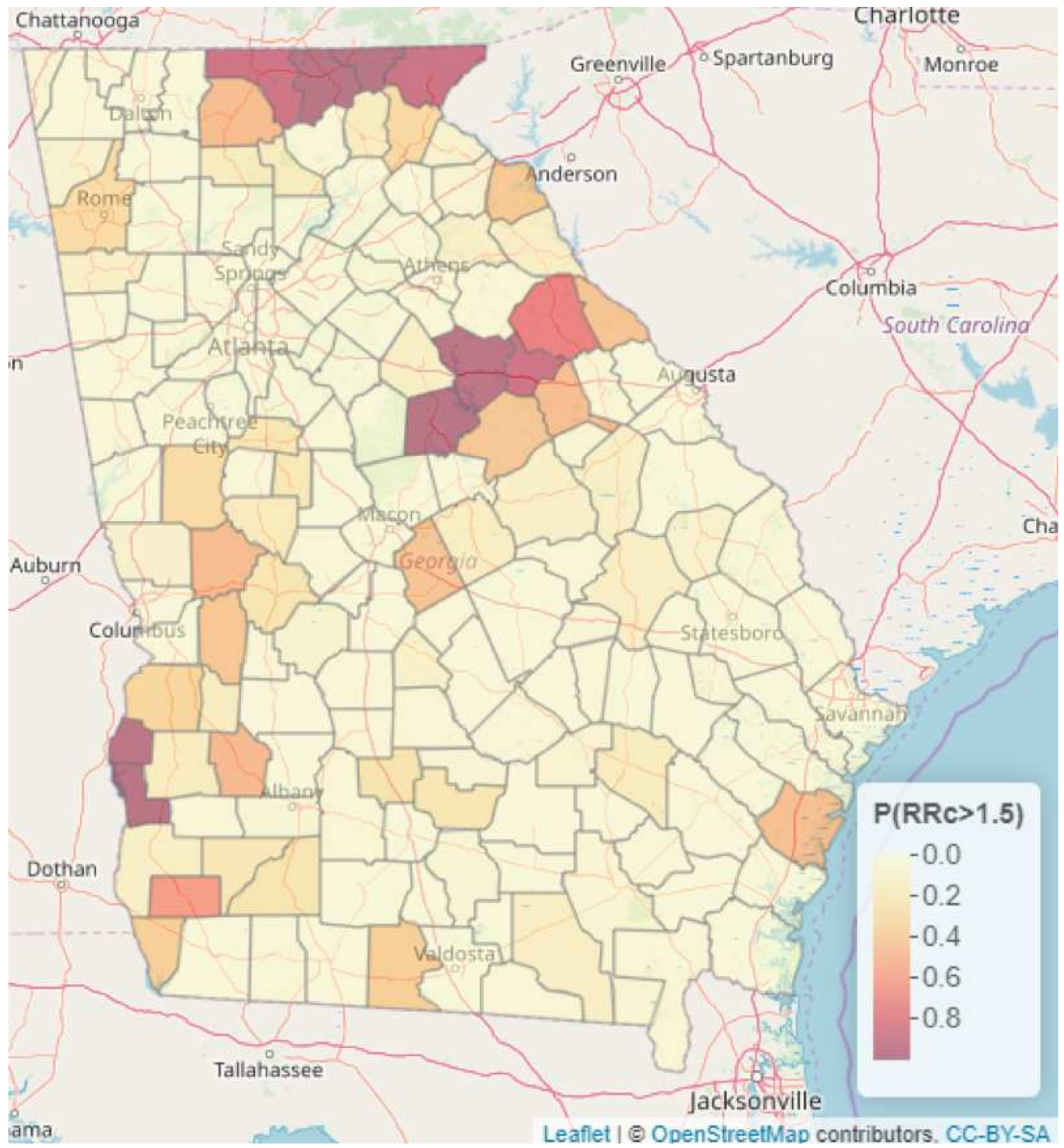

Figure 4

Predictive maps for exceedance probability of relative risk of 1.5 (i.e. $P(R R>1.5)$ ) 


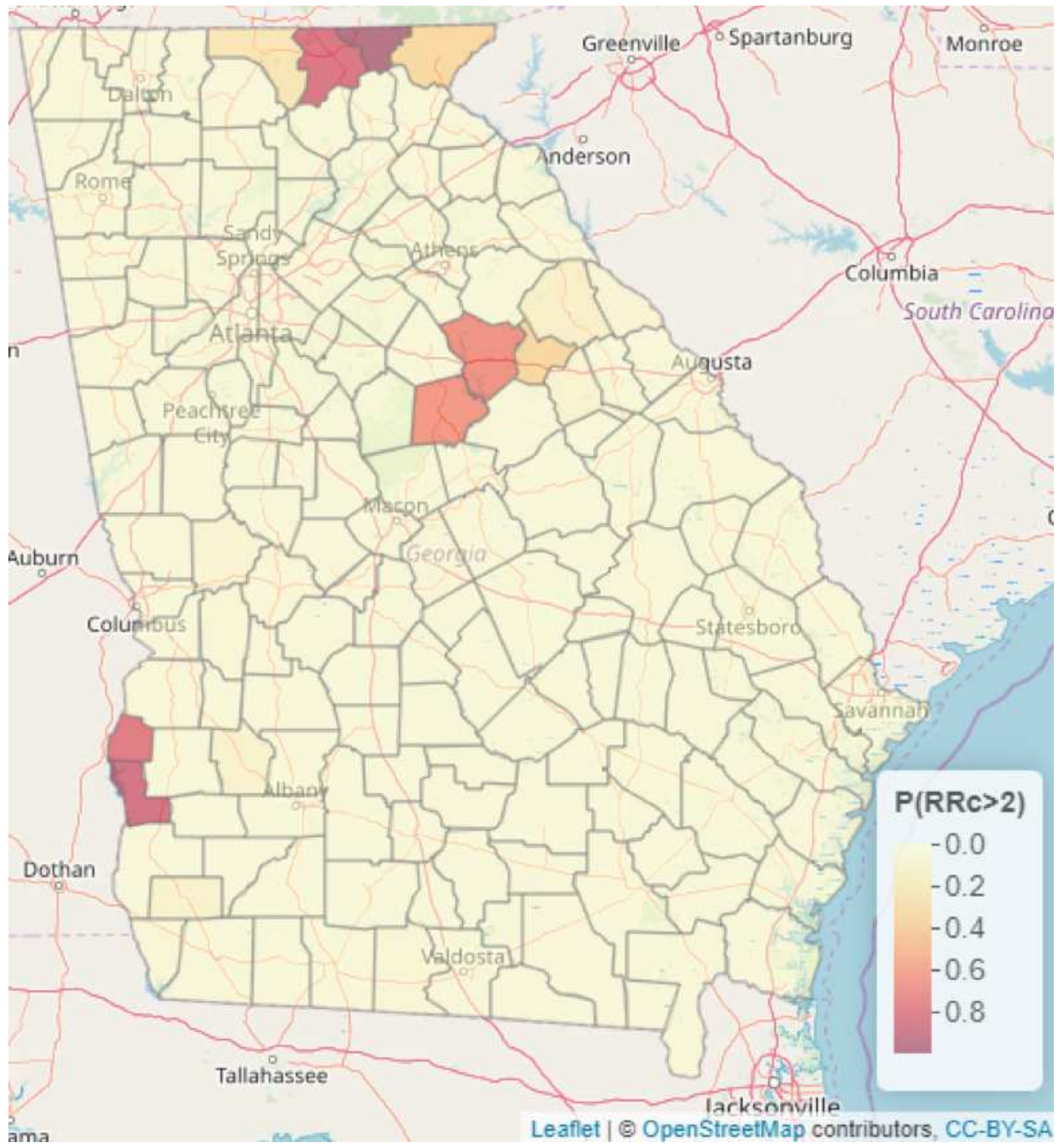

Figure 5

Predictive maps for exceedance probability of relative risk of 2 (i.e. $P(R R>2)$ )

\section{Supplementary Files}

This is a list of supplementary files associated with this preprint. Click to download. 
- Fig5.MapExcPRR2cWeb.html

- Fig4.MapExcPRR15cWeb.html

- Fig3.RRcMapsLeafletMalepopAll.html 